\title{
The Dynamics of Asset Sharing and Private Use
}

\author{
Thomas A. Weber \\ École Polytechnique Fédérale de Lausanne \\ thomas.weber@epfl.ch
}

\begin{abstract}
The collaborative consumption of an asset, such as a car, an appliance, or a power tool, tends to degrade it faster than when it is kept for purely personal use. This paper examines the rational dynamic decision of when to share an asset and when to use it only privately. An optimal policy trades off additional degradation and resulting lifetime reduction against the additional revenue from sharing. Solving the underlying continuous-time optimal control problem, we characterize three possible regimes: personal consumption, full sharing, and partial sharing. Collaborative consumption may be optimal only at the beginning of the asset's lifetime; the optimal time to switch from sharing to pure private consumption is obtained in closed form.
\end{abstract}

\section{Introduction}

Electronic markets for collaborative consumption have attained liquidity for assets that are intrinsically shareable. They have rendered the cost (including possible intermediary commissions) for sharing transactions small relative to a borrower's use value for the needed item, such as a power tool or a car. Given an effective price for sharing (net of transaction costs), we consider the question of how the extra degradation of assets from collaborative use, over and above the degradation expected from private use, determines individuals' intertemporal consumption and sharing decisions.

\subsection{Literature}

The possibility of collaborative consumption $[4,5,7]$ helps decouple individuals' incentives for owning assets from their (random) needs of using those same assets, clearing the path from an ownership-economy to an access-economy $[1,3,10]$. Despite the archetypal nature of sharing as a reciprocal activity which accompanied the development of mankind, electronic sharing markets have come into existence only about a decade ago. Reasons for this somewhat belated development include that only recently have intermediaries found ways to resolve informational asymmetries and match potential sharing partners sufficiently well, so that the perURI: http://hdl.handle.net/10125/50538

ISBN: 978-0-9981331-1-9

(CC BY-NC-ND 4.0) ceived costs for short-term sharing transactions would not exceed the expected gains from trade. A key informational asymmetry in the context of sharing comes from the moral hazard related to the unobservability of the borrower's actions, which may lead to a suboptimal effort to exert sufficient care when using the lender's asset. With the help of appropriate insurance contracts a trusted intermediary can mitigate moral hazard, provided the parties are sufficiently risk-neutral [16]. Reputation systems [14] have an important additional role to play by increasing the cost of borrower misbehavior in terms of rating loss and the resulting decreased ability to become a trusted counterparty in future sharing transactions. Here we ignore informational asymmetries and turn our attention to the fact that sharing an asset tends to degrade it faster-even in the absence of moral hazard-which has not yet been considered in the literature. Virtually any product has a finite lifetime, due to early failure, random failure, or wear and tear [2].

Our model uses a continuous-time approximation for an agent's dynamic consumption choice problem, which can be investigated using an optimal control analysis framework [17]. Because of the inherently linear structure of the relevant Hamiltonian in the control, the solution exhibits bang-bang switching behavior, as in the classical examples provided in the seminal text by Pontryagin et al. [11]. Switching between known deterministic cash-flow (or utility) streams was recently considered by Weber [15] using global-optimization methods. In our context, classical optimal-control theory can be used to obtain a closed form of the solution to the agents' switching problem, the solution of which is related to the classical Hotelling model for the extraction of a nonrenewable resource, which in our context corresponds to product lifetime in the absence of maintenance investments (see footnote 3 ).

\subsection{Outline}

The remainder of this paper is organized as follows. Sec. 2. introduces the model for an agent's dynamic sharing and personal-consumption decisions. Sec. 3. establishes the dynamic-choice behavior by solving the agent's optimal control problem. In Sec. 4., we discuss comparative statics of the solution. Sec. 5. concludes.

$H \stackrel{\text { "I }}{T} \mathrm{CSS}$ 


\section{Model}

We consider a single risk-neutral agent who owns a shareable asset. At each moment in time $t \geq 0$, the agent needs the asset with probability $\theta \in[0,1]$. In other words, the agent's random need states $\tilde{s}_{t}$, with realizations in $\{0,1\},{ }^{1}$ are independent and identically distributed according to

$$
\operatorname{Prob}\left(\tilde{s}_{t}=1\right)=\theta, \quad t \geq 0 .
$$

With this simplifying assumption we employ a continuous-time approximation, in the sense that while the length time for which the asset is needed is always infinitesimally short, over any extended time interval $\left[t_{0}, t_{1}\right)$ of nonzero measure (so $0 \leq t_{0}<t_{1}<\infty$ ), the aggregate time the agent would expect to need the asset is $\theta\left(t_{1}-t_{0}\right)$. When available for personal use the agent's instantaneous value of the asset at time $t$, conditional on a positive need $\left(s_{t}=1\right)$, is $V_{1}=v>0$. Without a need for the asset $\left(s_{t}=0\right)$, the agent's instantaneous value for it is $V_{0}=0$. The agent discounts future payoffs at the continuous discounting rate $r>0$. In other words, a dollar arriving at time $t>0$ is worth $e^{-r t}$ to the agent, the discount thus reflecting the time-value of money.

\subsection{Personal Use}

The agent's expected discounted value from purely personal use over the interval $\left[0, T_{P}\right]$ is

$$
\begin{aligned}
U_{P} & =\int_{0}^{T_{P}} e^{-r t}\left((1-\theta) V_{0}+\theta V_{1}\right) d t \\
& =\frac{\theta v}{r}\left(1-e^{-r T_{P}}\right),
\end{aligned}
$$

where the asset is enjoyed over its personalconsumption lifetime $T_{P}$. The smallest upper bound for the asset's lifetime value in personal consumption is the value of the perpetuity $\theta v$ over the interval $[0, \infty)$, so $U_{P} \leq \theta v / r$.

\subsection{Collaborative Consumption}

We assume that there is a peer-to-peer sharing market, where the asset can be offered at the effective price $p$. This price includes any commissions charged by a sharing intermediary as well as transaction costs which may arise when realizing the gains from collaborative consumption. $^{2}$ The agent is a price taker, and to exclude the trivial case where renting the item out on a sharing market is even better than using it when there is a need, we assume - without loss of generality - that

$$
p<v
$$

At any time $t$, the agent would therefore share the item only if there is no personal need for it. Hence, the agent's expected discounted value from sharing the asset when it is not needed, in the interval $\left[0, T_{S}\right]$, can be written in the form

$$
\begin{aligned}
U_{S} & =\int_{0}^{T_{S}} e^{-r t}((1-\theta) p+\theta v) d t \\
& =\frac{(1-\theta) p+\theta v}{r}\left(1-e^{-r T_{S}}\right),
\end{aligned}
$$

where $T_{S}$ is the (pure) sharing-lifetime of the asset.

\subsection{Asset-Degradation Dynamics}

The asset's lifetime depends on the nature of its use. The current degradation of the asset at time $t$ is represented by the asset's maintenance state $x(t)$ with values in the state space $\mathcal{X}=[0,1] .^{3}$ We allow the agent to dynamically decide about participation in the sharing market at time $t$, conditional on the maintenance state of the asset. The agent therefore chooses the control variable $u(t)$ with values in the control-constraint set $\mathcal{U}=\{0,1\}$, where $u(t)=0$ indicates personal consumption (i.e., no sharing) and $u(t)=1$ denotes collaborative consumption (i.e., sharing conditional on $s_{t}=0$ ) at time $t$.

When in personal use the asset degrades at the constant rate $\lambda>0$, while it degrades at the possibly elevated rate $\mu \geq \lambda$ when it is not used by the owner. ${ }^{4}$ As a result, the asset-degradation dynamics are given by the state equation

$$
\dot{x}(t)=-((1-\theta) \mu u(t)+\theta \lambda) x(t), \quad x(0)=x_{0},
$$

for $t \geq 0$. The positive initial state $x_{0}$ is normalized to 1 , without any loss of generality (by a linear change of units if necessary). Because the right-hand side of the preceding ordinary equation is always negative (taking into account the initial condition), the asset's maintenance state is strictly decreasing over time (see also footnote 3 ). The asset is fully degraded as soon as it reaches the discard threshold $x_{d} \in(0,1)$. Hence, for any admissible control $u$ the agent might choose, ${ }^{5}$ the

\footnotetext{
${ }^{1}$ At time $t \geq 0$, the agent has no need for the asset when $s_{t}=0$ and a need for the asset when $s_{t}=1$.

${ }^{2}$ The effective price can also include a less-than-perfect transaction probability as in Razeghian and Weber [13].

${ }^{3}$ To avoid unnecessary complications we exclude the possibility of a costly maintenance intervention and refurbishment, which would require additional assumptions about the cost and the effectiveness of such actions, distracting from our main concern, namely the dynamic tradeoff between personal and collaborative consumption.

${ }^{4}$ We focus here on wear and tear corresponding to the convex part of the classical "bathtub curve" for the hazard over the product lifecycle (see, e.g., [9], p. 27), although in practice the hazard-rate dynamics may well be more complicated [8]. In our simple setting, the time of product "failure" is perfectly known because the asset's maintenance state $x(t)$ attains the discard threshold $x_{d}$ at the deterministic time $t=T$.
}

${ }^{5}$ By "admissible control" we mean a measurable mapping $u: \mathbb{R}_{+} \rightarrow \mathcal{U}$. 
endogenous lifetime $T$ of the asset is implicitly specified by the terminal condition

$$
x(T)=x_{d} .
$$

Pure personal consumption and full asset sharing, described in Secs. 2.1 and 2.2, correspond to optimal controls of $u(t) \equiv 0$ and $u(t) \equiv 1$, respectively. The endogenous lifetime $T$ is therefore bracketed by the lifetimes under these two extreme modes of consumption, i.e.,

$$
T_{S} \leq T \leq T_{P}
$$

for any admissible control $u$ the agent might choose.

\section{Dynamic Choice}

\subsection{Agent's Control Problem}

Given a dynamic choice $u(t)$, for $t \in[0, T]$, the agent's expected discounted payoff is

$$
\Pi(u, T)=\int_{0}^{T} e^{-r t}(\theta p+(1-\theta) \varphi u) d t
$$

The agent's optimal control problem is therefore

$$
\Pi(u, T) \longrightarrow \max _{u(\cdot), T}
$$

subject to

$$
\begin{gathered}
\dot{x}=-(\theta \lambda+(1-\theta) \mu u) x, \quad x(0)=1, \quad x(T)=x_{d}, \\
u(t) \in \mathcal{U}, \quad t \in[0, T] .
\end{gathered}
$$

This corresponds to a Lagrange problem in the calculus of variations, with endpoint constraint and free endtime, to which a solution exists [6].

\subsection{Optimality Conditions}

In order to solve the agent's optimal control problem, we first introduce the current-value Hamiltonian

$H(x, u, \nu)=\theta v+(1-\theta) p u-\nu(\theta \lambda+(1-\theta) \mu u) x$,

where $\nu:[0, T] \rightarrow \mathbb{R}$ denotes an (absolutely continuous) adjoint variable. The adjoint variable describes the current value of the change in the maintenance state. The Hamiltonian represents the current dynamic use value of the asset (in collaborative and/or private consumption), including the value of the prevailing degradation momentum. Applying Pontryagin's maximum principle we obtain the following system of necessary optimality conditions $[11,17]$.

1. Maximality. On an optimal state-control trajectory the Hamiltonian needs to be maximal with respect to the control variable. Because $H$ is linear in $u$, the optimal choice of the control variable can be characterized by first determining the agent's indifference on the switching curve $\nu=\sigma(x)$, which satisfies the relation

$$
\mathcal{H}(x, 0, \sigma(x))=\mathcal{H}(q, 1, \sigma(x))
$$

where

$$
\mathcal{H}(x, 0, \sigma(x))=\theta v-\sigma(x) \theta \lambda x
$$

and

$\mathcal{H}(x, 1, \sigma(x))=\theta v+(1-\theta) p-\sigma(x)(\theta \lambda+(1-\theta) \mu) x$

Thus,

$$
\sigma(x)=\frac{p}{\mu x} \quad\left(\geq \frac{p}{\mu x_{d}}\right)
$$

For $\nu \geq \sigma(x)$ it is best to not share $(u=0)$, while for $\nu \leq \sigma(x)$ it is optimal to offer the item for sharing $(u=1)$.

2. Adjoint equation. The adjoint equation provides the law of motion for the adjoint variable $\nu$ :

$$
\dot{\nu}=r \nu-\mathcal{H}_{x}(x, u, \nu)=(r+\theta \lambda+(1-\theta) \mu u) \nu .
$$

Because of the state-endpoint constraint $x(t)=x_{d}$, a transversality condition for the endpoint $\nu(T)$ of the adjoint variable is not available. From the preceding law of motion (adjoint equation) it is clear that $\nu$ cannot change sign, so that necessarily $\nu(0)>0$. In addition, we know that $\nu(t)$ is exponentially increasing, thus leading to at most a single switch in the owner's sharing policy, from $u=1$ (use collaboratively) to $u=0$ (use alone). The initial arc, for $u=1$, up to the switching time $\tau \in[0, T]$ is given by

$$
\nu(t)=\nu(0) \exp [(r+\theta \lambda+(1-\theta) \mu) t], \quad t \in[0, \tau],
$$

while the final arc beyond the switching time is

$$
\nu(t)=\nu(\tau) \exp [(r+\theta \lambda)(t-\tau)], \quad t \in[\tau, T],
$$

with

$$
\nu(\tau)=\nu(0) \exp [(r+\theta \lambda+(1-\theta) \mu) \tau]
$$

A simplified description of the final arc is therefore

$\nu(t)=\nu(0) \exp [(1-\theta) \mu \tau+(r+\theta \lambda) t], \quad t \in[\tau, T]$.

3. Endpoint optimality. Because the asset lifetime $T$ is endogenous and subject to optimization, it needs to satisfy the endpoint optimality condition $\mathcal{H}(x(t), u(T), \nu(T))=0$. 


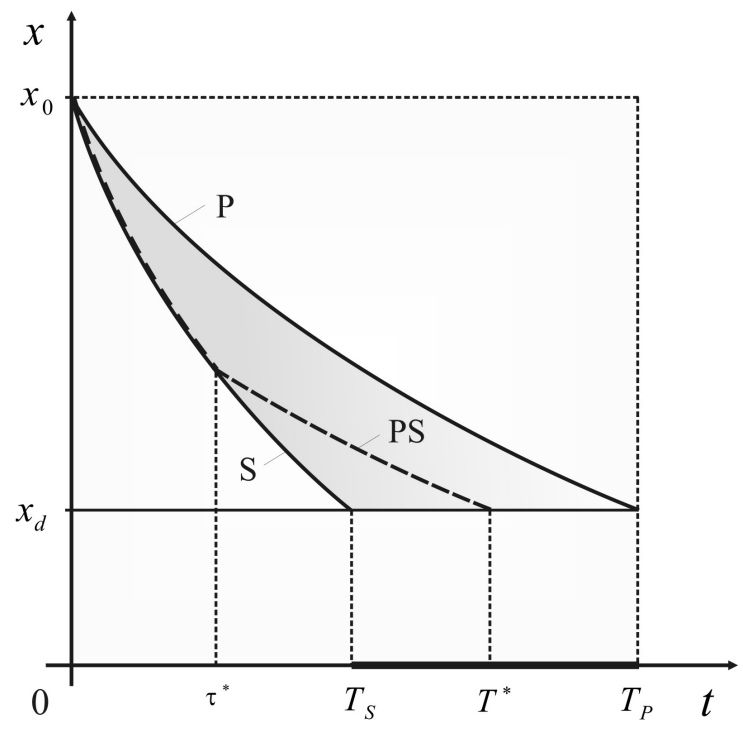

Figure 1: Optimal degradation dynamics.

Depending on the problem parameters, there can be three potentially optimal operating regimes: personal consumption $(\mathrm{P})$ corresponding to no sharing, full sharing (S), or partial sharing (PS); see Fig. 1.

\subsubsection{Regime P: Personal Consumption}

In the no-sharing regime, the optimal switching time is $\tau^{*}=0$, i.e., $u^{*}(t) \equiv 0$ is optimal for all $t \in[0, T]$. Thus,

$$
x(t)=x_{d}=\exp [-\theta \lambda T],
$$

which implies the longest possible optimal asset lifetime,

$$
T_{P}=\frac{\ln \left(1 / x_{d}\right)}{\theta \lambda}
$$

The corresponding optimal payoff is

$$
\Pi_{P}=\Pi\left(0, T_{P}\right)=\frac{\theta v}{r}\left[1-\left(x_{d}\right)^{\frac{r}{\theta \lambda}}\right] .
$$

\subsubsection{Regime S: Full Sharing}

In the full-sharing regime, the optimal switching time is $\tau^{*}=T$, i.e., $u^{*}(t) \equiv 1$ is optimal for all $t \in[0, T]$. Hence,

$$
x(t)=x_{d}=\exp [-(\theta \lambda+(1-\theta) \mu) T],
$$

which implies the shortest possible optimal asset lifetime,

$$
T_{S}=\frac{\ln \left(1 / x_{d}\right)}{\theta \lambda+(1-\theta) \mu}=\frac{\theta \lambda T_{P}}{\theta \lambda+(1-\theta) \mu} .
$$

The corresponding optimal payoff is

$$
\Pi_{S}=\Pi\left(0, T_{S}\right)=\frac{\theta v+(1-\theta) p}{r}\left[1-\left(x_{d}\right)^{\frac{r}{\theta \lambda+(1-\theta) \mu}}\right] .
$$

\subsubsection{Regime PS: Partial Sharing}

Consider now interior switching times $\tau \in(0,1)$. Because of the monotonicity of the adjoint variable in the preceding optimality conditions, several switches can never be optimal. The operator therefore starts out offering the asset on the sharing market in off-times and at some point takes it from the sharing market altogether. By the definitions of the switching time and the switching curve $\sigma$, it is

$$
\begin{aligned}
\nu(\tau) & =\sigma(x(\tau))=\frac{p}{\mu x(\tau)} \\
& =\nu(0) \exp [(r+\theta \lambda+(1-\theta) \mu) \tau] .
\end{aligned}
$$

The initial arc of the asset state is

$$
x(t)=\exp [-(\theta \lambda+(1-\theta) \mu) t], \quad t \in[0, \tau],
$$

while the final arc is given by

$$
\begin{aligned}
x(t) & =x(\tau) \exp [-\theta \lambda(t-\tau)] \\
& =\exp [-(1-\theta) \mu \tau-\theta \lambda t], \quad t \in[\tau, T]
\end{aligned}
$$

with

$$
x(\tau)=\exp [-(\theta \lambda+(1-\theta) \mu) \tau] .
$$

Hence, at the end of the horizon it is

$$
x_{d}=x(t)=\exp [-(1-\theta) \mu \tau-\theta \lambda T],
$$

so

$$
\begin{aligned}
T & =\frac{\ln \left(1 / x_{d}\right)-(1-\theta) \mu \tau}{\theta \lambda} \\
& =T_{P}-\left(\frac{1-\theta}{\theta}\right)\left(\frac{\mu}{\lambda}\right) \tau .
\end{aligned}
$$

Furthermore, the endpoint optimality implies

$$
0=\mathcal{H}\left(x_{d}, 0, \nu(T)\right)=\theta v-\nu(T) \theta \lambda x_{d},
$$

i.e.,

$$
\nu(T)=\frac{v}{\lambda x_{d}} .
$$

Using the earlier description of the final arc of the adjoint variable yields

$$
\begin{aligned}
\nu(T) & =\frac{v}{\lambda x_{d}}=\nu(0) \exp [(1-\theta) \mu \tau+(r+\theta \lambda) T] \\
& =\nu(0) e^{\frac{(r+\theta \lambda) \ln \left(1 / x_{d}\right)-(1-\theta) \mu r \tau}{\theta \lambda}} .
\end{aligned}
$$

Therefore

$$
\nu(0)=\left(\frac{v}{\lambda x_{d}}\right) e^{-\frac{(r+\theta \lambda) \ln \left(1 / x_{d}\right)-(1-\theta) \mu r \tau}{\theta \lambda}} \cdot \text { Page } 5208
$$


Taking into account the fact that $\nu(\tau)=\sigma(x(\tau))$, the preceding relation yields the switching time. For this, note that

$$
\begin{aligned}
\nu(\tau) & =\frac{v}{\lambda} \frac{e^{-\frac{(r+\theta \lambda) \ln \left(1 / x_{d}\right)-(1-\theta) \mu r \tau}{\theta \lambda}+(r+\theta \lambda+(1-\theta) \mu) \tau}}{x_{d}} \\
& =\frac{v e^{\left(1+\frac{r}{\theta \lambda}\right) \ln \left(x_{d}\right)+\left(\theta \lambda+(1-\theta) \mu+\left(1+\frac{(1-\theta) \mu}{\theta \lambda}\right) r\right) \tau}}{\lambda x_{d}} \\
& =\frac{v}{\lambda} \cdot\left(x_{d}\right)^{\frac{r}{\theta \lambda}} \cdot e^{\left(\theta \lambda+(1-\theta) \mu+\left(1+\frac{(1-\theta) \mu}{\theta \lambda}\right) r\right) \tau}
\end{aligned}
$$

and recall

$$
x(\tau)=\exp [-(\theta \lambda+(1-\theta) \mu) \tau],
$$

so

$$
\sigma(x(\tau))=\left(\frac{p}{\mu}\right) \exp [(\theta \lambda+(1-\theta) \mu) \tau] .
$$

We therefore obtain the optimality condition

$$
\frac{p}{\mu}=\frac{v}{\lambda} \frac{e^{-\left(1+\frac{r}{\theta \lambda}\right) \ln \left(\frac{1}{x_{d}}\right)+\left(1+\frac{(1-\theta) \mu}{\theta \lambda}\right) r \tau}}{x_{d}},
$$

which yields the optimal switching time

$$
\begin{aligned}
\tau^{*} & =\frac{\frac{\theta \lambda}{r} \ln \left(\frac{\lambda p}{\mu v}\right)+\ln \left(\frac{1}{x_{d}}\right)}{\theta \lambda+(1-\theta) \mu} \\
& =\frac{\theta \lambda}{\theta \lambda+(1-\theta) \mu}\left(T_{P}+\frac{\ln \left(\frac{\lambda p}{\mu v}\right)}{r}\right) \\
& =T_{S}-\frac{\theta \lambda}{\theta \lambda+(1-\theta) \mu}\left[\frac{1}{r} \ln \left(\frac{\mu v}{\lambda p}\right)\right] .
\end{aligned}
$$

Using the expression for the asset's lifetime $T$ as a function of the switching time $\tau$, together with the relation between $T_{S}$ and $T_{P}$ in Sec. 3.2.2., one therefore obtains the optimal asset lifetime under partial sharing:

$$
\begin{aligned}
T^{*} & =\frac{\theta \lambda T_{P}+(1-\theta) \mu\left[\frac{1}{r} \ln \left(\frac{\mu v}{\lambda p}\right)\right]}{\theta \lambda+(1-\theta) \mu} \\
& =T_{S}+\frac{(1-\theta) \mu}{\theta \lambda+(1-\theta) \mu}\left[\frac{1}{r} \ln \left(\frac{\mu v}{\lambda p}\right)\right] .
\end{aligned}
$$

And, the optimal payoff is given by:

$$
\begin{aligned}
\Pi^{*} & =\frac{\theta v}{r}\left(1-e^{-r T^{*}}\right)+\frac{(1-\theta) p}{r}\left(1-e^{-r \tau^{*}}\right) \\
& =\frac{\theta v+(1-\theta) p}{r}-\frac{(\theta \lambda+(1-\theta) \mu) p}{\mu r} e^{-r \tau^{*}} \\
& =\frac{\theta v+(1-\theta) p}{r}-A,
\end{aligned}
$$

where

$$
A=\frac{(\theta \lambda+(1-\theta) \mu) p}{\mu r}\left(\left(x_{d}\right)^{r}\left(\frac{\mu v}{\lambda p}\right)^{\theta \lambda}\right)^{\frac{1}{\theta \lambda+(1-\theta) \mu}} .
$$

In this we have used the fact that the time the asset is taken off the sharing market can be expressed in the compact form

$$
T^{*}-\tau^{*}=\frac{1}{r} \ln \left(\frac{\mu v}{\lambda p}\right)
$$

This "personal-use time" is positive if and only if

$$
\frac{p}{v}<\frac{\mu}{\lambda}
$$

Otherwise, the item is always offered for sharing during its downtime so as to maximize its utilization.

\subsection{Optimal Dynamic Sharing Policy}

In order to characterize the agent's optimal dynamic sharing policy we need to find conditions on the parameters for the relevance of regime P, S, or PS in the optimal solution. For this, we first compare the "boundary regimes" ( $\mathrm{P}$ and $\mathrm{S}$ ) and then characterize the situations where the "interior regime" (PS) is best for the decisionmaker.

\subsubsection{Boundary Regimes: $P$ and $S$}

Comparing regimes $\mathrm{P}$ and $\mathrm{S}$, it is clear that full sharing dominates personal consumption if and only if

$$
(1-\theta) p\left[1-\left(x_{d}\right)^{\frac{r}{\theta \lambda+(1-\theta) \mu}}\right]
$$

does exceed

$$
\theta v\left[\left(x_{d}\right)^{\frac{r}{\theta \lambda+(1-\theta) \mu}}-\left(x_{d}\right)^{\frac{r}{\theta \lambda}}\right],
$$

i.e., if and only if the expected revenue from collaborative consumption exceeds the present value of the difference in usage value due to the shorter asset life induced by offering it on the sharing market.

\subsubsection{Interior Regime: PS}

We now examine the optimal switching time $\tau^{*}$ in regime PS. This switching time is positive if

$$
x_{d}<\left(\frac{\lambda p}{\mu v}\right)^{\theta \lambda / r}
$$

A sufficient condition is that the right-hand side of this inequality exceeds 1 , i.e.,

$$
\frac{p}{v}>\frac{\mu}{\lambda}
$$

Thus, if the ratio of sharing price to usage value $(p / v)$ is larger than the ratio of asset degradation with sharing to asset degradation without sharing $(\mu / \lambda)$, then sharing 
will be optimal on some nontrivial time interval. Otherwise, there is a threshold

$$
\bar{\theta}=\left(\frac{r}{\lambda}\right) \frac{\ln \left(\frac{1}{x_{d}}\right)}{\ln \left(\frac{\mu v}{\lambda p}\right)},
$$

so that $\theta<\bar{\theta}$ implies that sharing is optimal at least initially.

\subsubsection{Summary}

The interior partial-sharing regime (PS) is optimal if and only if

$$
\frac{p}{v}<\frac{\mu}{\lambda} \text { and } \theta<\bar{\theta}
$$

Finally, $\Pi_{P}>\Pi_{S}$ if and only if

$$
\frac{p}{v}>\frac{\theta}{1-\theta} \frac{\left(x_{d}\right)^{r /(\theta \lambda+(1-\theta) \mu)}-\left(x_{d}\right)^{r /(\theta \lambda)}}{1-\left(x_{d}\right)^{r /(\theta \lambda+(1-\theta) \mu)}} .
$$

The policy regions are depicted in Fig. 2. Note that if the own-degradation rate $\lambda$, the degradation threshold $x_{d}$ or the ratio $(\mu v) /(\lambda p)$ are sufficiently small, the threshold $\bar{\theta}$ exceeds 1 , so $\theta \in[0,1]$ can never exceed $\bar{\theta}$.

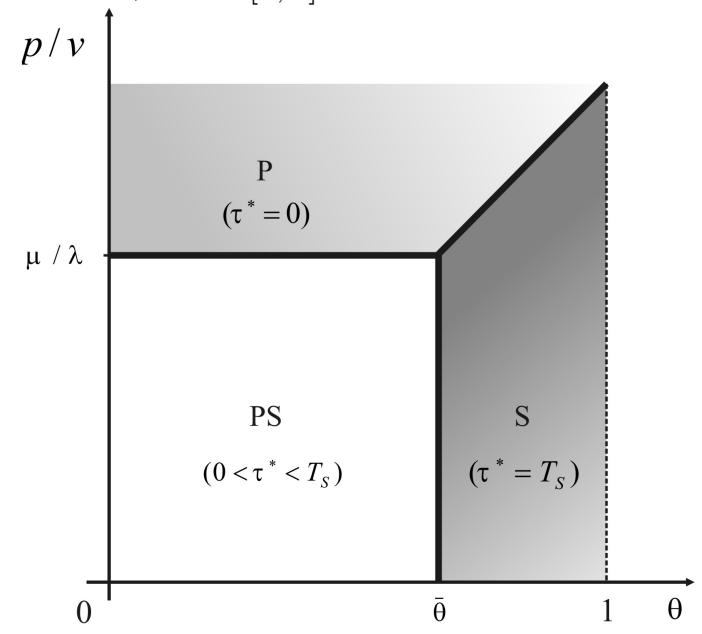

Figure 2: Agent's optimal dynamic consumption policy.

\section{Managerial Implications}

To draw conclusions for managerial decisions, it is useful to examine the sensitivity of the rational sharing behavior to key parameters.

\subsection{Comparative Statics of $\tau^{*}$}

Consider first the sensitivity of the optimal switching time under partial sharing; the latter corresponds to the time for which goods are rationally shared, thus minimizing the asset downtime. By straightforward differentiation one can show that the optimal switching time $\tau^{*}$ is increasing in the discount rate $r$ and the sharing price $p$. Hence, as agents become less concerned about the future or the sharing market promises greater economic rents, assets are used collaboratively for a longer time. On the other hand, the switching time is decreasing in the discard threshold $x_{d}$ and the contingent consumption value $v$. This means that less durable goods get shared for less time, and-similarly - an increase in the asset's need-contingent personal consumption value can only lead to less sharing. With respect to the likelihood of need one obtains that $\tau^{*}$ is decreasing in $\theta$ if and only if $-(\theta \lambda+(1-\theta) \mu)-(\bar{\theta}-\theta)(\lambda-\mu)<0$. This in turn is equivalent to $0<\bar{\theta} \lambda+(1-\bar{\theta}) \mu$, which is always satisfied. A larger personal utilization of the asset leads to a shorter collaborative-consumption time.

Regarding the effect of changes in the degradation rates, consider first an increase in the sharing-related elevated degradation constant $\mu$. The switching time $\tau^{*}$ decreases in $\mu$ if and only if

$$
-\frac{\theta}{\mu}(\theta \lambda+(1-\theta) \mu)-(1-\theta)(\bar{\theta}-\theta) \ln \left(\frac{\mu v}{\lambda p}\right)<0,
$$

which is always satisfied in the interior of the partial-sharing regime. Hence, an increase of the degradation from collaborative consumption leadsunsurprisingly - to a shorter time of sharing.

Finally, the monotonicity of the switching on the "natural" degradation from renting is not definite, and depends on the parameters. Indeed, $\tau^{*}$ is increasing in $\lambda$ if and only if

$$
(\theta \lambda+(1-\theta) \mu)\left(\frac{1}{\ln \left(\frac{\mu v}{\lambda p}\right)}-1\right)>(\bar{\theta}-\theta) \lambda .
$$

For example, the inequality does not hold when $p$ becomes small enough. In that case, the switching time decreases in $\lambda$. The reason for the sign-ambiguity is that $\lambda$ is important both when the asset is shared and when it is not shared.

\subsection{Comparative Statics of $\Pi^{*}$}

Consider first the boundary regimes of personal consumption (P) and full sharing (S). One can show that $\Pi_{P}$ is increasing in $\theta / r$ and $v$, and that it is decreasing in $x_{d}$ and $\lambda$. One can also show that $\Pi_{S}$ is increasing in $v$ and $p$, and that it is decreasing in $x_{d}, r, \mu$, and $\lambda$. The comparative statics of $\Pi^{*}$ are more complicated. It is clear that it is decreasing in $x_{d}$ and decreasing in $r$.

\subsection{Comparative Statics of Lifetimes}

Let $\Delta=T_{P}-T_{S}$ be the diameter of the set of attainable lifetimes. Then $\Delta$ is decreasing in $x_{d}, \lambda / \mu$, and $\theta$. Furthermore, because $T_{P} \rightarrow \infty$ as $\theta \rightarrow 0^{+}$while $T_{P}$ 
stays bounded, we also obtain that $\Delta \rightarrow \infty$ as $\theta \rightarrow 0^{+}$. This means that when personal utilization tends to zero, the agent can make the asset last an arbitrarily long time by withholding it from the peer-to-peer sharing market.

\section{Conclusion}

The economic optimality of sharing does not just depend on whether an item is idle at any given time. Our analysis shows that the lifetime value of an asset is generally not optimized by maximizing its utilization, as it is sometimes suggested in the literature on sharing. Because increased utilization decreases its lifetime, the sharing price must be sufficiently large to justify collaborative consumption, even in the absence of moral hazard. The second-order consequence of sharing markets is to influence product durability because more highly used assets degrade faster. ${ }^{6}$ The solution to the agent's dynamic decision problem shows that sharing is a beginning-of-life phenomenon: collaboratively used products are fairly new. This means that at relatively low maintenance state of the product individuals may become reluctant to share, which therefore decreases the end-of-life utilization of assets. The latter would be further compounded by impending replacement costs, which were not considered in this paper. Embedding the optimal sharing decisions into a larger product-replacement problem remains an interesting topic for future research. In addition, there is a set of activities concerned with extending the product life (e.g., through refurbishments and repairs), which deserves more detailed investigation in the context of individuals' asset-acquisition and use decisions.

\section{References}

[1] Bardhi, F., Eckhardt, G.M. (2012) "AccessBased Consumption: The Case of Car Sharing," Journal of Consumer Research, Vol. 39, No. 4, pp. 881-898.

[2] Barlow, R.E., Proschan, F. (1975) Statistical Theory of Reliability and Life Testing: Probability Models, Holt, Rinehart \& Winston, New York, NY.

[3] Belk, R. (2014). "You Are What You Can Access: Sharing and Collaborative Consumption online," Journal of Business Research, Vol. 67, No. 8, pp. 1595-1600.

[4] Benkler, Y. (2004) "Sharing Nicely: On Shareable Goods and the Emergence of Sharing as a Modality of Economic Production," Yale Law Journal, Vol. 114, No. 2, pp. 273-358.
[5] Botsman, R., Rogers, R. (2010) What's Mine Is Yours: How Collaborative Consumption Is Changing the Way We Live, HarperCollins, London, UK.

[6] Cesari, L. (1983) Optimization: Theory and Applications, Springer, New York, NY.

[7] Felson, M., Spaeth, J.L. (1978) "Community Structure and Collaborative Consumption: A Routine Activity Approach,' American Behavioral Scientist, Vol. 21, No. 4, pp. 614-624.

[8] Klutke, G.-A., Kiessler, P.C., Wortman, M.A. (2003) "A Critical Look at the Bathtub Curve," IEEE Transactions on Reliability, Vol. 52, No. 1, pp. 125-129.

[9] Nelson, W. (1982) Applied Life Data Analysis, Wiley, New York, NY.

[10] NiELSEN (2014) "Is Sharing the New Buying?" Nielsen Company, New York, NY, May 28.

[11] Pontryagin, L.S., Boltyanskit, V.G., GAMKRElidZE, R.V., MishCHENKO, E.F. (1962) The Mathematical Theory of Optimal Processes, Wiley Interscience, New York, NY.

[12] Razeghian, M., Weber, T.A. (2016) "The Effect of Sharing Markets on Product Durability," Working Paper, Ecole Polytechnique Fédérale de Lausanne, Lausanne, Switzerland.

[13] Razeghian, M., Weber, T.A. (2015) "To Share or Not to Share: Adjustment Dynamics in Sharing Markets," Working Paper, Ecole Polytechnique Fédérale de Lausanne, Lausanne, Switzerland.

[14] Resnick, P., Kuwabara, K., Zeckhauser, R., Friedman, E. (2000) "Reputation Systems," Communications of the ACM, Vol. 43, No. 12, pp. $45-48$.

[15] Weber, T.A. (2017) "Optimal Switching Between Cash-Flow Streams," Mathematical Methods of Operations Research, forthcoming.

[16] Weber, T.A. (2014) "Intermediation in a Sharing Economy: Insurance, Moral Hazard, and Rent Extraction," Journal of Management Information Systems, Vol. 31, No. 3, pp. 35-71.

[17] Weber, T.A. (2011) Optimal Control Theory with Applications in Economics, MIT Press, Cambridge, MA.

\footnotetext{
${ }^{6}$ For the effect of sharing markets on firm's incentives to provide product durability, see Razeghian and Weber [12].
} 